The performance of the examination is closely monitored by the Royal College of Psychiatrists' Examinations Sub-Committee with robust quality assurance processes in place. The content and performance of each item is scrutinised pre- and post-examination. The College is also required to provide data and reports to the regulator (the General Medical Council, GMC) and any proposed changes to the examination require GMC's approval. Recent changes approved by the GMC include a reduction from three written papers to two (introduced from this year) and a change to the CASC marking scheme from the Hofstee method to borderline regression (from diet 2 this year). As part of the process to reduce the number of written papers, the written paper question banks have been fully reviewed and updated. The statement that MCQs are continuously recycled year after year is incorrect. New questions are constantly being developed and every examination paper has about $40 \%$ of new questions. All questions have been mapped to the examinations syllabus and new question writing is focused on areas of the question bank where the range of questions is limited. There is also a focus on developing a greater range of questions testing clinical management within Paper B.

The MRCPsych examination is under continuous review and development by the Examinations Sub-Committee. An external review of the examinations was commissioned in 2014 and we are following up on recommendations for further enhancements to the MRCPsych. These are due to be published at the end of 2015 .

The curriculum, like the examination, is under constant review in a process that involves a wide community including lay people, trainees, medical managers, psychiatry experts and trainers. All changes have to be approved by the GMC and there is regular dialogue between the College and the GMC. A major revision of the core curriculum is being planned and will include the incorporation of the examination syllabus.

While we understand that trainees may feel the MRCPsych is another hurdle, ultimately, the College is responsible for ensuring that quality and patient safety are at the forefront of its examination processes. We are satisfied that the current standard is appropriate for entry into higher training. While it is our ambition to drive up the standard, we are aware that a significant proportion of core trainees struggle to achieve the standards set by the examination. The College is keen to influence training and the learning experience of trainees. To this end we have introduced Trainees Online (TrOn; http://tron.rcpsych.ac.uk), a series of online learning modules for trainees that will eventually cover the whole MRCPsych examination syllabus. We have also been working with MRCPsych course organisers to improve the standard and consistency of courses. We hope that increased clarity about what trainees need to know will lead to higher examination pass rates as well as the acquisition of knowledge that will support clinical practice.

Dr Wendy Burn, Dean, and Dr Peter Bowie, Chief Examiner, Royal College of Psychiatrists, London, email: c/o pb@rcpsych.ac.uk

doi: $10.1192 /$ pb.39.5.262a

\section{Psychiatry is more than neuropsychiatry}

In his editorial, Fitzgerald ${ }^{1}$ rehashes the well-trodden arguments for the reunification of neurology and psychiatry, suggesting the time has finally come. What he fails to address is that the trend in every sphere of medicine is towards further specialisation and not integration. Why psychiatry and neurology should be the exception to the rule goes unanswered.

It is only ever academic psychiatrists, appearing out of touch with clinical practice, who propose that psychiatry has advanced to the point where it is indistinguishable from neurology. On the contrary, despite the calls for psychiatry to become a clinical neuroscience discipline, ${ }^{2}$ psychiatric practice has remained untouched by developments in neuroscience. To be sure, neuroscience is a core basic science for psychiatry. But the claims that psychiatric disorders are simply brain disorders, or that our observations or interventions are not worth a jot if not based in neuroscience, are part of a creeping trend towards neuroessentialism in every sphere of life. ${ }^{3}$ Psychiatrists do not simply deal with brain disorders - to claim otherwise is to impoverish our field. Psychiatry is at its best when embracing a pluralistic approach to the disparate range of problems that fall under our gaze. To neglect insights from the psychological, sociological and anthropological sciences and the narrative approach to formulation does a disservice to our patients. The patient who becomes suicidal after a relationship breakdown and the patient who becomes panic-stricken and housebound after a rape do not have problems that can be made sense of in the same way as the patient with visual hallucinations and bradykinesia, or the patient with impulse control problems after a brain injury. Put simply, even if we accept the claim that psychiatric problems are brain disorders, many problems can be effectively treated without thinking about the brain.

Psychiatrists could certainly benefit from a stronger training in clinical neuroscience and neurology in general, and neuropsychiatry and behavioural neurology in particular. But as Alwyn Lishman said, 'You have got to have a finger in every pie in psychiatry and be ready to turn your hand to whatever is the most important avenue: an EEG one day, a bit of talking about a dream another day. You just follow your nose. All psychiatrists should be all types of psychiatrist'. ${ }^{4}$ I could not agree more.

Vivek Datta, Chief Resident, Department of Psychiatry and Behavioral Sciences, University of Washington Medical Center, Seattle, USA, email: vdatta@mail.harvard.edu

1 Fitzgerald M. Do psychiatry and neurology need a close partnership or a merger? BJPsych Bull 2015; 39: 105-7.

2 Insel TR, Quirion R. Psychiatry as a clinical neuroscience discipline. JAMA 2005; 294: 2221-4.

3 Reiner PB. The Rise of Neuroessentialism. In The Oxford Handbook of Neuroethics (eds J Iles, B Sahakian): 161-75. Oxford University Press, 2011.

4 Poole NA. Interview with Professor William Alwyn Lishman. Psychiatrist 2013; 37: 343-4.

doi: 10.1192/pb.39.5.263

\section{A more practicel solution is needed}

Professor Fitzgerald is worried about the serious recruitment crisis in psychiatry. His answer is to advise psychiatrists to abandon their specialty and 'return home to neurology'. In his opinion, a merger of the two professions would encourage clinicians to focus on careful clinical analysis and diagnosis, 